International Journal of Pure and Applied Mathematics

Volume 105 No. $3 \quad$ 2015, 383-392

ISSN: 1311-8080 (printed version); ISSN: 1314-3395 (on-line version)

url: http://www.ijpam.eu

doi: http://dx.doi.org/10.12732/ijpam.v105i3.6

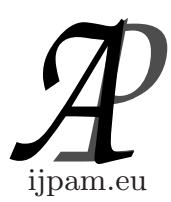

\title{
SCHULTZ AND GUTMAN INDICES FOR GRAPH COMPLEMENTS
}

\author{
S. Ramakrishnan ${ }^{1 \S}$, J. Baskar Babujee ${ }^{2}$ \\ ${ }^{1}$ Department of Mathematics \\ Sri Sai Ram Engineering College \\ Chennai 600044, INDIA \\ ${ }^{2}$ Department of Mathematics \\ Anna University \\ Chennai 600025, INDIA
}

\begin{abstract}
A graph $G$ is said to have property $(*)$ [4] if for every pair of its adjacent vertices $u$ and $v$ there exists a vertex $w$ such that $w$ is not adjacent to $u$ and $v$. In this paper we establish an explicit formula to calculate the Schultz and Gutman indices for the complement of any graph $G$ having the above property. As a corollary we obtain the Schultz and Gutman indices for the complement of certain derived graphs.
\end{abstract}

AMS Subject Classification: 05C10, 05C12, 92E10, 94C15

Key Words: Gutman index, line graph, Schultz index, subdivision graph, Zagreb index

\section{Introduction}

A graph $G$ consists of a set of vertices $V$ and a set of edges $E$. If the vertices $u$ and $v$ are connected by an edge $e$ we denote it by $e=(u, v)$. The degree of a vertex $u$ denoted by $\operatorname{deg}_{G}(u)$ represents the number of edges incident to it and $d_{G}(u, v)$ denote the distance between the vertices $u$ and $v$ and is the length of

Received: July 29, 2015

(C) 2015 Academic Publications, Ltd.

$\S$ Correspondence author url: www.acadpubl.eu 
the shortest path connecting $u$ and $v$. If the graph $G$ is connected, then the greatest distance between two of its vertices is the diameter of $G$, denoted by $\operatorname{diam}(G)$. The complement of a graph $G$ denoted by $\bar{G}$ is the graph with the same vertex set as that of $G$, in which two vertices are adjacent if and only if they are not adjacent in $G$. A topological index of a graph $G$ is the graph invariant number calculated from a graph representing a molecule. The Wiener index is one of the oldest and most studied topological indices both from the theoretical point of view and applications. It is equal to the sum of distances between all pairs of vertices of the corresponding graph. In this paper we are concerned with the invariants of connected graphs namely Schultz and Gutman indices. In 1989, H.P. Schultz [7] introduced a new topological index, namely Schultz index which is defined as $S(G)=\sum_{\{u, v\} \subseteq V}\left(\operatorname{deg}_{G}(u)+\operatorname{deg}_{G}(v)\right) d_{G}(u, v)$. Andrey A. Dobrynin and Amide A. Kochetova in 1994 [1] also proposed the above index and called it the degree distance of a graph. Motivated by the Schultz index, S. Klavžar and I. Gutman introduced Schultz index of the second kind in 1997 called Gutman index [5]. Gutman index of a graph $G$ is defined as $G u t(G)=$ $\sum_{\{u, v\} \subseteq V}\left(\operatorname{deg}_{G}(u) \operatorname{deg}_{G}(v)\right) d_{G}(u, v)$. J. Senbagamalar, J. Baskar Babujee and Ivan Gutman [4] have characterized certain classes of graphs possessing property $(*)$ (see Definition 1.7) and established the Wiener index of graph complements. In this paper we compute the Schultz and Gutman indices of graph complements for the classes of graphs possessing property $(*)$. The present paper is organized as follows. In Section 2 definitions and results required for main results are introduced. In Section 3 we establish an explicit formula to calculate the Schultz and Gutman indices of the complement of a graph $\mathrm{G}$ and use them to find the complements of certain derived graphs.

\section{Preliminaries}

In what follows we consider a simple connected undirected graph $G$ with vertex set $V$ and edge set $E$.

Definition 1. [6] The first and second Zagreb indices of $G$ denoted by $M_{1}(G)$ and $M_{2}(G)$ respectively are defined as:

$$
M_{1}(G)=\sum_{u \in V}\left[\operatorname{deg}_{G}(u)\right]^{2}, M_{2}(G)=\sum_{(u, v) \in E} \operatorname{deg}_{G}(u) \operatorname{deg}_{G}(v) \text { where } \operatorname{deg}_{G}(u)
$$

denotes the degree of the vertex $u$ in $G$.

Definition 2. [8] The hyper-Zagreb index of the graph $G$ is defined as 


$$
H M(G)=\sum_{(u, v) \in E}\left[\operatorname{deg}_{G}(u)+\operatorname{deg}_{G}(v)\right]^{2} .
$$

We now define certain graphs which are derived from the graph $G$.

Definition 3. [3] Line graph of $G$ denoted by $L$ is the graph whose vertices correspond to the edges of $G$ with two vertices in $L$ being adjacent iff the corresponding edges in $G$ are incident.

$$
|V(L)|=|E|,|E(L)|=\frac{1}{2} M_{1}(G)-|E| .
$$

Definition 4. [3] Subdivision graph of $G$ is the graph $S$ obtained from $G$ by inserting a new vertex into each edge of $G$.

$$
|V(S)|=|V|+|E|,|E(S)|=2|E| .
$$

Definition 5. [3] Edge-semi total graph of $G$ is the graph $T_{2}$ obtained from $G$ by inserting a new vertex into each edge of $G$ and by joining edges to those pairs of these new vertices which lie on adjacent edges of $G$.

$$
\left|V\left(T_{2}\right)\right|=|V|+|E|,\left|E\left(T_{2}\right)\right|=|E|+\frac{1}{2} M_{1}(G) .
$$

Definition 6. [3] Vertex-semi total graph of $G$ is the graph $T_{1}$ obtained from $G$ by adding a new vertex corresponding to each edge of $G$ and by joining each new vertex to the end vertices of the edge corresponding to it.

$$
\left|V\left(T_{1}\right)\right|=|V|+|E|,\left|E\left(T_{1}\right)\right|=3|E| .
$$

Definition 7. [3] Total graph of $G$ is the graph $T(G)$ whose vertex set is $V \cup E$, with two vertices of $T(G)$ being adjacent if and only if the corresponding elements of $G$ are adjacent or incident.

$$
|V(T)|=|V|+|E|,|E(T)|=2|E|+\frac{1}{2} M_{1}(G) .
$$

Definition 8. [4] The graph $G$ is said to have property (*) if for every pair of its adjacent vertices $u$ and $v$, there exists a third vertex $w$ which is not adjacent to either $u$ or $v$. If $G$ has property $\left(^{*}\right)$, then $\bar{G}$ is connected and $\operatorname{diam}(\bar{G})=2$.

In what follows we assume that $G=(V, E)$ is a connected graph with $|V|=n$ and $|E|=m$. 
Lemma 9. [3, 9] The first Zagreb indexes of some graphs which are derived from $G$ (refer Definitions 3 - 7) are:

(i) $M_{1}(L)=F-4 M_{1}(G)+2 M_{2}(G)+4 m$ where

$$
F=\sum_{(u, v) \in E}\left[\operatorname{deg}_{G}(u)^{2}+\operatorname{deg}_{G}(v)^{2}\right]
$$

(ii) $M_{1}(S)=M_{1}(G)+4 m$

(iii) $M_{1}\left(T_{1}\right)=4 M_{1}(G)+4 m$

(iv) $M_{1}\left(T_{2}\right)=M_{1}(G)+M_{1}(L)+8|E(L)|+4 m$

(v) $M_{1}(T)=4 M_{1}(G)+M_{1}(L)+8|E(L)|+4 m$

Lemma 10. The second Zagreb indexes of some derived graphs of $G$ are:

(i) $M_{2}(S)=2 M_{1}(G)$

(ii) $M_{2}\left(T_{1}\right)=4 M_{2}(G)+4 M_{1}(G)$

(iii) $M_{2}(T)=4 M_{2}(G)+2 H M(G)+M_{2}(L)+2 M_{1}(L)+2 M_{1}(G)-4 m$

(iv) $M_{2}\left(T_{2}\right)=H M(G)+M_{2}(L)+2 M_{1}(L)+2 M_{1}(G)-4 m$

Proof.

(i)

$$
\begin{aligned}
M_{2}(S) & =\sum_{(u, v) \in E(S)} \operatorname{deg}_{S}(u) \operatorname{deg}_{S}(v) \\
& =\sum_{(u, v) \in E(G)}\left[2\left(\operatorname{deg}_{G}(u)\right)+2\left(\operatorname{deg}_{G}(v)\right)\right]=2 M_{1}(G)
\end{aligned}
$$

(ii)

$$
\begin{aligned}
M_{2}\left(T_{1}\right)= & \sum_{(u, v) \in E\left(T_{1}\right)} \operatorname{deg}_{T_{1}}(u) \operatorname{deg}_{T_{1}}(v) \\
= & \sum_{(u, v) \in E(G)}\left(2 \operatorname{deg}_{G}(u)\right)\left(2\left(\operatorname{deg}_{G}(v)\right)\right. \\
& \quad+\sum_{(u, v) \in E(G)}\left[2\left(2 \operatorname{deg}_{G}(u)\right)+2\left(2\left(\operatorname{deg}_{G}(v)\right)\right]\right. \\
= & 4\left(M_{2}(G)+M_{1}(G)\right)
\end{aligned}
$$


(iii)

$$
\begin{aligned}
M_{2}(T)= & \sum_{(u, v) \in E(T)} \operatorname{deg}_{T}(u) \operatorname{deg}_{T}(v) \\
= & \sum_{(u, v) \in E(G)}\left(2 \operatorname{deg}_{G}(u)\right)\left(2\left(\operatorname{deg}_{G}(v)\right)\right. \\
& +2 \sum_{(u, v) \in E(G)}\left[\begin{array}{c}
{\left[\left(\operatorname{deg}_{G}(u)+\operatorname{deg}_{G}(v)\right) \operatorname{deg}_{G}(u)\right.} \\
+\left(\operatorname{deg}_{G}(u)+\operatorname{deg}_{G}(v)\right) \operatorname{deg}_{G}(v)
\end{array}\right] \\
& +\sum_{(u, v) \in E(L)}\left[\left(\operatorname{deg}_{L}(u)+2\right)\left(\operatorname{deg}_{L}(v)+2\right)\right] \\
M_{2}(T)= & 4 M_{2}(G)+2 H M(G)+M_{2}(L)+2 M_{1}(L)+2 M_{1}(G)-4 m .
\end{aligned}
$$

(iv)

$$
\begin{aligned}
M_{2}\left(T_{2}\right)= & \sum_{(u, v) \in E\left(T_{2}\right)} \operatorname{deg}_{T_{2}}(u) \operatorname{deg}_{T_{2}}(v) \\
= & \sum_{(u, v) \in E(G)}\left[\begin{array}{c}
\left(\operatorname{deg}_{G}(u)+\operatorname{deg}_{G}(v)\right) \operatorname{deg}_{G}(u) \\
+\left(\operatorname{deg}_{G}(u)+\operatorname{deg}_{G}(v)\right) \operatorname{deg}_{G}(v)
\end{array}\right] \\
& +\sum_{(u, v) \in E(L)}\left[\left(\operatorname{deg}_{L}(u)+2\right)\left(\operatorname{deg}_{L}(v)+2\right)\right] \\
= & H M(G)+M_{2}(L)+2 M_{1}(L)+2 M_{1}(G)-4 m .
\end{aligned}
$$

\section{Main Results}

Theorem 11. If $G$ has property $\left({ }^{*}\right)$ then

(i) the Schultz index of $\bar{G}$ is given by $S(\bar{G})=n(n-1)^{2}-M_{1}(G)$

(ii) the Gutman index of $\bar{G}$ is given by

$$
\operatorname{Gut}(\bar{G})=\frac{1}{2}\left[(n-1)^{2}\left(n^{2}-n-2 m\right)+4 m^{2}-(2 n-1) M_{1}(G)+2 M_{2}(G)\right]
$$

Proof.

(i)

$$
S(\bar{G})=\sum_{\{u, v\} \subseteq V(\bar{G})}\left(\operatorname{deg}_{\bar{G}}(u)+\operatorname{deg}_{\bar{G}}(v)\right) d_{\bar{G}}(u, v)
$$




$$
\begin{aligned}
= & \sum_{e=(u, v) \in E(G)}\left(\left((n-1)-\operatorname{deg}_{G}(u)\right)+\left((n-1)-\operatorname{deg}_{G}(v)\right)\right)(2) \\
& +\sum_{e=(u, v) \notin E(G)}\left(\left((n-1)-\operatorname{deg}_{G}(u)\right)+\left((n-1)-\operatorname{deg}_{G}(v)\right)\right)(1) \\
= & 4 m(n-1)-2 \sum_{e=(u, v) \in E(G)}\left[\operatorname{deg}_{G}(u)+\operatorname{deg}_{G}(v)\right] \\
& +2\left(\frac{n(n-1)}{2}-m\right)(n-1)-\sum_{e=(u, v) \notin E(G)}\left[\operatorname{deg}_{G}(u)+\operatorname{deg}_{G}(v)\right] \\
= & 4 m(n-1)-2 M_{1}(G)+(n-1)\left(n^{2}-n-2 m\right)-\bar{M}_{1}(G) \\
= & 2 m(n-1)+n(n-1)^{2}-2 M_{1}(G)-2 m(n-1)+M_{1}(G) \\
= & n(n-1)^{2}-M_{1}(G) .
\end{aligned}
$$

(ii)

$$
\begin{aligned}
G u t(\bar{G})= & \sum_{\{u, v\} \subseteq V(\bar{G})}\left(\operatorname{deg}_{\bar{G}}(u) \operatorname{deg}_{\bar{G}}(v)\right) d_{\bar{G}}(u, v) \\
= & \sum_{e=(u, v) \in E(G)}\left(\left((n-1)-\operatorname{deg}_{G}(u)\right)\left((n-1)-\operatorname{deg}_{G}(v)\right)\right)(2) \\
& +\sum_{e=(u, v) \notin E(G)}\left(\left((n-1)-\operatorname{deg}_{G}(u)\right)\left((n-1)-\operatorname{deg}_{G}(v)\right)\right)(1) \\
= & 2 m(n-1)^{2}-2(n-1) M_{1}(G)+2 M_{2}(G) \\
& +\left(\frac{n(n-1)}{2}-m\right)(n-1)^{2}-(n-1) \bar{M}_{1}(G)+\bar{M}_{2}(G) \\
= & (n-1)^{2}\left(\frac{n(n-1)}{2}+m\right)-2(n-1) M_{1}(G) \\
& -(n-1)\left(2 m(n-1)-M_{1}(G)\right) \\
& +2 M_{2}(G)+\left(2 m^{2}-M_{2}(G)-\frac{1}{2} M_{1}(G)\right) \\
= & \frac{1}{2}\left[(n-1)^{2}\left(n^{2}-n-2 m\right)+4 m^{2}-(2 n-1) M_{1}(G)+2 M_{2}(G)\right] .
\end{aligned}
$$

Corollary 12. Let $n \geq 5$, and let $T$ be a tree of order $n$ which is neither the star nor a double star. Then $S(\bar{T})=n(n-1)^{2}-M_{1}(T)$ and

$$
G u t(\bar{T})=\frac{1}{2}\left[(n-1)^{2}\left(n^{2}-3 n+6\right)-(2 n-1) M_{1}(T)+2 M_{2}(T)\right]
$$


Proof. As $R$ has property (*) for $n \geq 5, S(\bar{T})$ and $G u t(\bar{T})$ are obtained by replacing $m$ by $n-1$ in Theorem 11 (i) and (ii) respectively.

Corollary 13. Let $T$ be a $r$-regular graph of order $n$. If $r \leq(n-1) / 2$, then $S(\bar{R})=n\left((n-1)^{2}-r^{2}\right)$ and

$$
\operatorname{Gut}(\bar{R})=\frac{1}{2}\left[n(n-r-1)\left((n-1)^{2}-r^{2}\right)\right]=\frac{1}{2}(n-r-1) S(\bar{R}) .
$$

Proof. As $R$ has property $(*)$ if $r \leq(n-1) / 2$, we obtain $S(\bar{R})$ and $\operatorname{Gut}(\bar{R})$ substituting $m=\frac{1}{2} n r$ in Theorem 11(i) and (ii) respectively.

Corollary 14. Let $C_{n}$ be a cycle of order $n$. If $n \geq 5$, then $S\left(\overline{C_{n}}\right)=$ $n\left((n-1)^{2}-4\right)=n(n+1)(n-3)$ and

$$
\operatorname{Gut}\left(\overline{C_{n}}\right)=\frac{1}{2}\left[n(n-3)^{2}(n+1)\right] \text {. }
$$

Proof. As $C_{n}$ is a special case of the regular graph $R$ of order $n$ and degree 2, $S\left(\overline{C_{n}}\right)$ and $G u t\left(\overline{C_{n}}\right)$ are obtained by replacing $r=2$ in $S(\bar{R})$ and $G u t(\bar{R})$ respectively.

Corollary 15. Let $G^{+}$be the graph obtained by attaching a pendent vertex to each vertex of $G$. If $G$ is connected and $n \geq 2$ then

$$
\begin{gathered}
S\left(\overline{G^{+}}\right)=8 n^{2}(n-1)-4 m-M_{1}(G) \\
G\left(\overline{G^{+}}\right)=\frac{1}{2}\left[8 n^{2}\left(2(n-1)^{2}-m\right)+4 m(m+2)-(4 n-3) M_{1}(G)+2 M_{2}(G)\right] .
\end{gathered}
$$

Proof. As $G^{+}$has property $(*)$ with $2 n$ vertices and $(m+n)$ edges, we obtain from Theorem 11 (i) and (ii),

$$
\begin{gathered}
S\left(\overline{G^{+}}\right)=2 n(2 n-1)^{2}-M_{1}\left(G^{+}\right), \\
\operatorname{Gut}\left(\overline{G^{+}}\right)=\frac{1}{2}\left[(2 n-1)^{2}\left(4 n^{2}-2 n-2(m+n)\right)+4(m+n)^{2}\right. \\
\left.-(4 n-1) M_{1}\left(G^{+}\right)+2 M_{2}\left(G^{+}\right)\right] .
\end{gathered}
$$

Since $M_{1}\left(G^{+}\right)=M_{1}(G)+4 m+2 n$ and $M_{2}\left(G^{+}\right)=M_{1}(G)+M_{2}(G)+3 m+n$, we get on simplifying $S\left(\overline{G^{+}}\right)=8 n^{2}(n-1)-4 m-M_{1}(G)$ and

$$
\begin{aligned}
\operatorname{Gut}\left(\overline{G^{+}}\right)=\frac{1}{2}\left[8 n^{2}\left(2(n-1)^{2}-m\right)+4 m(\right. & m+2) \\
& \left.-(4 n-3) M_{1}(G)+2 M_{2}(G)\right] .
\end{aligned}
$$


Corollary 16. Let $S$ be the subdivision graph of $G$ such that $G$ such that $G \neq P_{n}, n \leq 2$ then

$$
S(\bar{S})=(n+m)(n+m-1)^{2}-4 m-M_{1}(G)
$$

and

$$
\begin{aligned}
G u t(\bar{S})=\frac{1}{2}\left[(n+m)(n+m-1)^{3}+4 m\left(4 m-(m+n)^{2}\right)\right. & \\
& \left.-M_{1}(G)(2 n+2 m-5)\right] .
\end{aligned}
$$

Proof. $S$ has property $(*)$ with $(n+m)$ vertices and $2 m$ edges and hence using Theorem 11(i) and (ii) together with Lemma 9 and Lemma 10 we get

$$
S(\bar{S})=(n+m)(n+m-1)^{2}-4 m-M_{1}(G)
$$

and

$$
\begin{aligned}
\operatorname{Gut}(\bar{S})=\frac{1}{2}\left[(n+m)(n+m-1)^{3}+4 m(4 m-\right. & \left.(m+n)^{2}\right) \\
& \left.-M_{1}(G)(2 n+2 m-5)\right] .
\end{aligned}
$$

Corollary 17. Let $T_{1}$ be the vertex-semi total graph of $G$ (see Definition 6 ) and if $G \neq P_{n}$ for $n \leq 3, G \neq K_{n} \forall n$ and $G \neq K_{1, n}$ for $n \geq 3$ then

$$
S\left(\overline{T_{1}}\right)=(n+m)(n+m-1)^{2}-4 m-4 M_{1}(G)
$$

and

$$
\operatorname{Gut}\left(\overline{T_{1}}\right)=\frac{1}{2}\left[\begin{array}{c}
(n+m)(n+m-1)^{3}+2 m\left(18 m-3 m(m+n-1)^{2}\right. \\
-4(n+m)+2)-4 M_{1}(G)(2 n+2 m-3)+8 M_{2}(G)
\end{array}\right] .
$$

Proof. $T_{1}$ has property $(*)$ with $(n+m)$ vertices and $3 m$ edges and hence using Theorem 11 (i) and (ii) together with Lemma 9 and Lemma 10 we get the result.

Corollary 18. Let $T_{2}$ be the edge - semi total graph of $G$ (see Definition 5) and if $G \neq P_{n}, n \leq 3$ and $G \neq K_{n} \forall n$ then

$$
S\left(\overline{T_{2}}\right)=(n+m)(n+m-1)^{2}-M_{1}(G)-H M(G)
$$

and

$$
\operatorname{Gut}\left(\overline{T_{2}}\right)=\frac{1}{2}\left[\begin{array}{l}
(n+m-1)^{2}\left[(n+m)^{2}-(n+3 m)\right]+4 m(m-2) \\
+M_{1}^{2}(G)+2 M_{2}(L(G))-M_{1}(G)\left((n+m)^{2}\right. \\
-4(m+1))-H M(G)(2(n+m)-3)+4 M_{1}(L(G))
\end{array}\right] .
$$


Proof. $T_{2}$ has property $(*)$ with $n+m$ vertices and $\left(m+\frac{1}{2} M_{1}(G)\right)$ edges and hence using Theorem 11 (i) and (ii) together with Lemma 9 and Lemma 10 we get the result.

Corollary 19. Let $T$ be the total graph of $G$ (see Definition 7) and if $G \neq P_{n}$ for $n \leq 3, G \neq K_{n} \quad \forall n$ and $G \neq K_{1, n}$ for $n \geq 3$ then

$$
S(\overline{T(G)})=(n+m)(n+m-1)^{2}-4 M_{1}(G)-H M(G)
$$

and

$$
S^{*}(\overline{T(G)})=\frac{1}{2}\left[\begin{array}{l}
(n+m-1)^{2}\left[(n+m)^{2}-(n+5 m)\right]+8 m(2 m-1) \\
+M_{1}^{2}(G)-M_{1}(G)\left((n+m)^{2}+6 n-2 m-7\right) \\
-H M(G)(2(n+m)-1)+8 M_{2}(G) \\
+2 M_{2}(L(G))+4 M_{1}(L(G))
\end{array}\right] .
$$

Proof. $T(G)$ has property $(*)$ with $n+m$ vertices and $\left(2 m+\frac{1}{2} M_{1}(G)\right)$ edges and hence using Theorem 11 (i) and (ii) together with Lemma 9 and Lemma 10 we get the result.

Corollary 20. Let $L$ be the line graph of $G=(V, E)$ and if there exists no edge $(u, v) \in E$ of degree $(m-1)$ then

$$
\begin{gathered}
S(\bar{L})=m(m-1)^{2}-M_{1}(L), \\
\operatorname{Gut}(\bar{L})=\frac{1}{2}\left(m(m+1)(m-1)^{2}+\right. \\
+m^{2}-(m+1)^{2} M_{1}(G) \\
\left.+M_{1}^{2}(G)-(2 m-1) M_{1}(L)+2 M_{2}(L)\right) .
\end{gathered}
$$

Proof. If there exist no edge $(u, v) \in E$ of degree $(m-1)$ then the line graph possesses property $\left(^{*}\right)$ and hence using Theorem 11 and that fact $|V(L)|=$ $|E|=m$ and $|E(L)|=\frac{1}{2} M_{1}(G)-|E|$ we get the result.

\section{Conclusion}

In this paper formulae to compute Schultz and Gutman indices for the complement of graphs $G$ possessing property $(*)$ are established. Second Zagreb indices for derived graphs of $G$ namely subdivision, vertex - semi total, edge semi total and total graphs are also found and the results obtained are used to compute the above indices for the complement of the derived graphs of $G$. Similar results pertaining to various other topological indices for graph complements may be tried out. 


\section{References}

[1] Andrey A. Dobrynin, Amide A. Kochetova, Degree Distance of a Graph: A Degree analogue of the Wiener Index, J. Chem. Inf. Comput. Sci., 34 (1994), 1082-1086.

[2] J. Baskar Babujee, S. Ramakrishnan, Zagreb indices and coindices for compound graphs, In Mathematical and Computational Models, Narosa Publishing House, New Delhi (2012).

[3] Ivan Gutman, Boris Furtula, Žana Kovijanić Vukićević and Goran Popivoda, On Zagreb Indices and Coindices, MATCH Commun. Math. Comput. Chem., 74 (2015), 5-16.

[4] Jaisankar Senbagamalar, Jayapal Baskar Babujee and Ivan Gutman, On Wiener Index of Graph Complements, Transactions on Combinatorics, $\mathbf{3}$, No. 2 (2014), 11-15.

[5] S. Klavžar and I. Gutman, Wiener number of vertex-weighted graphs and a chemical application, Disc. Appl. Math., 80 (1997), 73-81.

[6] Nenad Trinajstić, Chemical Graph Theory, Volume II, CRC Press, Inc., Boca Raton, Florida (1983).

[7] H.P. Schultz, Topological Organic Chemistry 1, Graph Theory and Topological Indices of Alkanes, J. Chem. Inf. Comput. Sci., 29 (1989) 227-228.

[8] G.H. Shirdel, H. Rezapour and A.M. Sayadi, The hyper-Zagreb index of graph operations, Iranian Journal of Mathematical Chemistry, 4, No. 2 (2013), 213-220.

[9] M. Tavakoli, F. Rahbarnia, Note on properties of first Zagreb index of graph, Iranian Journal of Mathematical Chemistry, 3, No. 1 (2012), 51-55. 